Didáctica. Lengua y literatura

ISSN-e: 1988-2548

\title{
¿Para qué sirven los dictados? Representaciones de los futuros maestros de primaria
}

\author{
Jordi Cicres ${ }^{1}$; Sílvia Llach $^{2}$
}

Recibido: 6 de junio de 2017 / Aceptado: 30 de abril de 2019

Resumen. El objetivo de este trabajo es analizar los conocimientos y las creencias de los maestros de Enseñanza Primaria sobre el dictado, una actividad que ha sido objeto de diferentes visiones a través de distintas teorías de la enseñanza y el aprendizaje. Para ello, hemos diseñado un cuestionario compuesto por preguntas abiertas y lo hemos administrado a 150 estudiantes de segundo curso del grado de Maestro en Educación Primaria y del doble grado de Maestro en Educación Primaria y Maestro en Educación Infantil de la Universidad de Gerona (antes de realizar sus prácticas en las escuelas). Los resultados del cuestionario se han analizado mediante técnicas cualitativas y análisis estadístico de conglomerados jerárquicos. Los datos analizados revelan que la mayoría de los estudiantes solo conocen entre dos y tres dictados distintos, aunque algunos estudiantes llegan a mencionar hasta cinco. Considerando los usos del dictado, hemos observado que los estudiantes encuestados entienden que el uso predominante de esta actividad es mejorar o evaluar el conocimiento de la ortografía. Estos dos aspectos, los tipos y usos predominantes, también han sido agrupados por el análisis estadístico realizado. En relación con esto, el trabajo incluye recomendaciones finales para promover prácticas de dictado más variadas, para integrarlas de forma natural en prácticas pedagógicas coherentes de cada centro educativo, que estén de acuerdo con las visiones contemporáneas del aprendizaje. Palabras clave: dictados, escucha, representaciones, ortografía.

\section{[en] What are dictations useful for? Representations of future primary teachers}

\begin{abstract}
The aim of this paper is to analyse pre-service primary teachers' knowledge and beliefs about dictation, an activity that has been interpreted in different ways according to distinct theories of teaching and learning. In order to do this, we have designed a questionnaire composed of openended questions and have administered it to 150 students from the second course of the Primary-School Education degree and the double degree in Primary-School Education and Early Childhood Education at the University of Girona (before their practicum periods). The results of the questionnaire have been analysed using qualitative techniques and hierarchical clustering. Data revealed that most students only know between two and three different dictation methods, although some students mention up to five. Considering its uses, we have observed that the interviewed students understand that the predominant use of this activity is to improve or assess knowledge of spelling. These two aspects, the predominant types and uses, were also grouped in the statistical analysis. In relation to this, the paper presents final recommendations to foster more diverse dictation practices, and to integrate them in a natural way into the cohesive pedagogical practices of each school, in line with contemporary views of learning.
\end{abstract}

Keywords: dictations, listening, representations, spelling.

1 Institut de Recerca Educativa

Universitat de Girona

jordi.cicres@udg.edu

2 Institut de Recerca Educativa

Universitat de Girona

silvia.1lach@udg.edu 


\section{[fr] Dictée : usages et préconisations. Représentations des futurs enseignants de Primaire}

Résumé. L'objectif a été d'analyser les connaissances et les croyances des enseignants de Primaire en matière de dictée, une activité qui a fait objet de différentes visions selon une diversification de théories de l'enseignement et de l'apprentissage. Pour ce faire, nous avons conçu un questionnaire composé de questions ouvertes et nous l'avons ditribué à 150 étudiants du diplôme de deuxième cycle de l'enseignement en école primaire et du double diplôme de l'enseignement en école primaire et de l'éducation en petite enfance de l'Université de Gérone (avant leurs stages). Nous avons compilé les résultats du questionnaire en utilisant plusieurs techniques qualitatives et l'analyse statistique de conglomérés hiérarchiques. Le données recueillies révèlent que la plupart des étudiants connaissent seulement entre deux et trois types de dictées différents, même si certains étudiants en mentionnent jusqu'à cinq. Concernant son utilisation, nous avons observé que les étudiants interviewés comprennent que l'objectif d'une utilisation prédominante de cette activité est d'améliorer ou d'évaluer la connaissance de l'orthographe. Nous avons aussi regroupé ces deux aspects, les types et les usages prédominants, dans l'analyse statistique réalisée. Le résultat nous a conduits à formuler diverses préconisations finales afin de promouvoir des pratiques de la dictée plus variées, en les intégrant de façon naturelle dans les pédagogies cohérentes de chaque établissement éducatif, en accord avec les visions contemporaines de l'apprentissage.

Mots-clés: dictées, écoute, représentations, orthographe.

Sumario: 1. Introducción. 2. Los dictados en la educación primaria. 3. Las representaciones de los dictados. 3.1. Informantes, material y método. 3.2. Resultados. 4. Conclusiones y recomendaciones. Bibliografía

Cómo citar: Cicres, J.; Llach, S. (2019). ¿Para qué sirven los dictados? Representaciones de los futuros maestros de primaria, Didáctica. Lengua y literatura, 31, 47-63.

\section{Introducción}

En Uno, dos, tres, la película dirigida y producida por Billy Wilder en 1961, aparecen tres escenas hoy irreconocibles para la mayoría de jóvenes. Las tres están protagonizadas por G. R. McNamara (James Cagney), el director general de la sucursal de Coca-Cola en Berlín Occidental en 1961, y Fräulein Ingeborg (Liselotte Pulver), su secretaria. En las tres escenas el director la llama a su despacho para dictarle un texto (un informe mensual en la primera y un anuncio clasificado en el que se oferta un puesto de trabajo en las otras dos). La secretaria toma notas a mano en un bloc para, posteriormente, mecanografiarlo.

Estas escenas, en un momento en que la tecnología de reconocimiento del habla ha llegado a todos los bolsillos en forma de aplicaciones para teléfonos inteligentes, pueden parecer surrealistas a los niños y jóvenes. Además, en la actualidad prácticamente nadie dicta nada a nadie, sino que cada persona escribe sus textos directamente al procesador de textos. Existen, sin embargo, por lo menos dos ámbitos en los que los dictados sobreviven. Por un lado, en programas de televisión como El gran dictat ${ }^{3}$, que durante 7 temporadas (entre 2009 y

3 El concurso consiste en una serie de pruebas, entre las que encontramos una de deletrear palabras con los ojos tapados y la de escribir correctamente palabras de dificultad creciente (las del nivel más difícil son habitualmente palabras correspondientes al léxico especializado de disciplinas científicas diversas). 
2016) ha sumado más de 1200 emisiones en TV3 (la televisión pública catalana), con un promedio de 200.000 espectadores (y una cuota de pantalla del $11 \%$, con picos de audiencia de 300.000 espectadores y un $20,5 \%$ de share) y en el que han participado más de 2000 concursantes; o en actos de carácter simbólico, como el dictat en català (un acto conmemorativo del edicto del rey Luis XIV que prohibió en 1700 la lengua catalana en las comarcas catalanas bajo dominio francés desde 1659) que se celebra anualmente desde el año 2000 en Perpiñán como acto para reivindicar la lengua catalana en este territorio tradicionalmente catalanohablante del sur de Francia con notable éxito de participación, o la dictada occitana, que desde 1998 reivindica el uso del occitano, también prohibido por el edicto. Por el otro lado, los dictados siguen plenamente vigentes en el ámbito escolar, desde primaria hasta la ESO o incluso el Bachillerato (Cassany, 2004; Generalitat de Catalunya, 2014).

Esta práctica continuada se hace presente cuando se pregunta a estudiantes universitarios del grado de Maestro de Educación Primaria ${ }^{4}$ sobre actividades que consideran útiles para aprender lenguas en la Educación Primaria. Ante la propuesta «Haz una lista de entre 5 y 10 actividades que consideres adecuadas y útiles para aprender la primera lengua - catalán o castellano- en Primaria y justifica por qué», un 52\% citan los dictados como ejercicios que ayudan a los estudiantes a mejorar su ortografía, con matices (el más repetido es que copiar las faltas de ortografía diez veces es inútil). Sin embargo, en el mismo grupo de estudiantes, un porcentaje también significativo (el 24\%) considera que los dictados son actividades poco adecuadas para aprender lenguas (la pregunta era paralela a la anterior: "Haz una lista de entre 5 y 10 actividades que recuerdes haber realizado como alumno de Primaria o ESO que consideres inadecuadas o poco útiles para aprender la primera lengua -catalán o castellano-y justifica por qué").

En este artículo vamos a situar los dictados en la teoría sobre el proceso de enseñanza-aprendizaje de la lengua (apartado 2) y a analizar con detalle los preconocimientos y las representaciones que tienen los jóvenes estudiantes del grado de Maestro en Educación Primaria de la Universidad de Gerona sobre los dictados (apartado 3). Finalmente, en las conclusiones se aportan algunas orientaciones dirigidas a maestros en activo, a estudiantes de magisterio y a formadores en didáctica de la lengua para orientar en el uso de esta herramienta didáctica (apartado 4).

\section{Los dictados en la Educación Primaria}

El dictado es una práctica escolar habitual en la Educación Primaria y Secundaria que consiste en "comprender un texto oral y codificarlo al canal escrito", y mayoritariamente se utiliza con dos finalidades distintas: como una actividad de aprendizaje, centrada principalmente en la práctica de las reglas ortográficas, y como un instrumento de evaluación del dominio de la ortografía (Cassany, 2004, 231).

Stansfield (1985) hace un recorrido histórico por las vicisitudes de los dictados desde la Edad Media (cuando el maestro los utilizaba para transmitir los contenidos

4 Los estudiantes a los que se preguntó por escrito fueron los mismos que respondieron el cuestionario objeto de análisis en este trabajo. Las características de los encuestados se explican en el apartado 3.1. 
a los alumnos, o como la forma habitual para copiar los libros en los scriptoria) hasta la década de 1980, en la que los dictados gozaban de una buena aceptación. Entre tanto, la técnica ha pasado por altibajos. Por ejemplo, tanto el método natural ${ }^{5} \mathrm{como}$ el método de la respuesta física total para el aprendizaje de lenguas extranjeras descartaron el dictado como ejercicio válido en las clases, puesto que se priorizaba la enseñanza de la lengua ora ${ }^{6}$. Contrariamente, el enfoque basado en la gramática y la traducción (utilizado desde el siglo XVI) y el método directo consideraron el dictado como un ejercicio válido y especialmente útil, aunque por razones distintas: para el primer método, porque permitía practicar las reglas gramaticales y el vocabulario aprendidos; para el método directo, porque era un ejercicio para la enseñanza de la fonética.

A partir del siglo XX, los dictados se empiezan a utilizar como herramientas para evaluar los conocimientos en lengua (y específicamente en ortografía) de los alumnos, no sin polémica, por dos motivos. Por un lado, porque a priori los dictados son ejercicios que están desvinculados de la práctica lingüística real; por el otro, porque el dictado parece que tiene poco que ver con el dominio de las habilidades lingüísticas, como critica Lado $(1961,34)$ :

Dictation is favored by many teachers and students both as a teaching and testing device. However, on critical inspection it appears to measure very little of language. Since the order of words is given by the examiner as he reads the material, it does not test word order. Since the words are given by the examiner, it does not test vocabulary. It hardly tests aural perception of the examiner's pronunciation, because the words can in many cases be identified by context if the student does not hear the sounds correctly. The student is less likely to hear the sounds incorrectly in the slow reading of the words which is necessary for dictation. Spelling and a few matters of inflection and punctuation can be tested through dictation, but the complicated apparatus of dictation is not required to test such matters. Simpler techniques can be substituted.

Sin embargo, algunos estudios ya clásicos (como Valette, 1964; Oller, 1971, o Irvin, Atai \& Oller, 1974) encontraron una correlación entre el resultado obtenido en el dictado y el resultado global de las pruebas de dominio lingüístico (que incluían ejercicios gramaticales, de audición y de redacción). A fecha de hoy, el dictado se sigue utilizando como herramienta de evaluación, puesto que es un ejercicio complejo en el que actúan varios procesos parcialmente diferentes de los que se encuentran en la escritura espontánea. Cuetos $(2011,43-49)$ los esquematiza, y propone tres vías distintas para su escritura, que pueden combinarse según las situaciones de dictado y los conocimientos del escribiente (Figura 1):

\footnotetext{
5 Para una descripción de estos métodos de enseñanza de lenguas extranjeras, recomendamos la lectura de Richards \& Rodgers (2014) y Sánchez (2009).

6 Sin embargo, algunos autores como Joynes $(1900,28)$, citado por Stansfield $(1985,122)$, señalaron que el dictado era útil porque el canal visual reforzaba el aprendizaje del canal auditivo.
} 


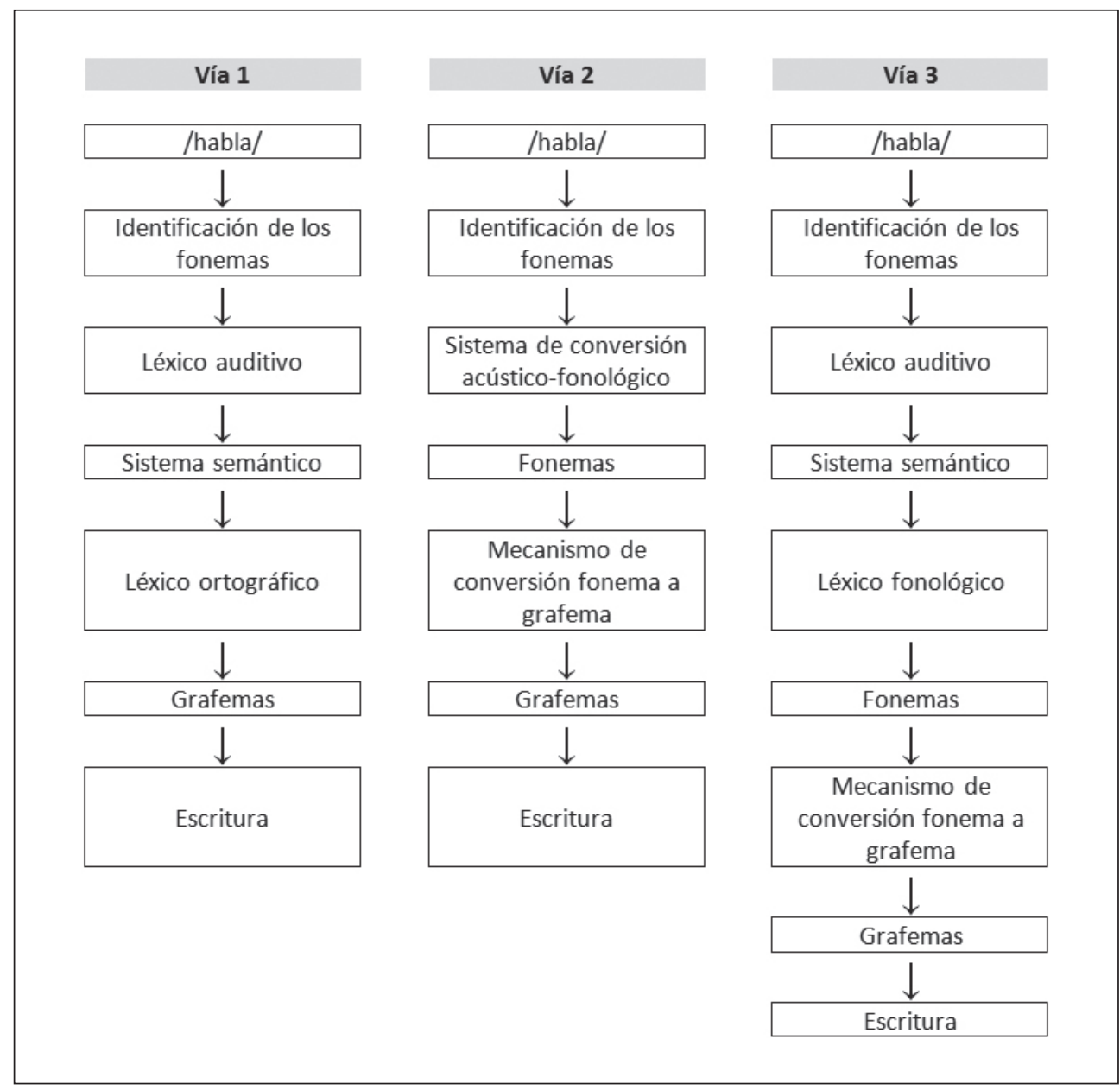

Figura 1. Procesos que intervienen en la escritura del dictado.

Fuente: Cuetos, F. (2011). Psicología de la escritura ( $8^{a}$ edición).

Las Rozas, Madrid: Wolters Kluwer España, 44 (adaptado).

En todas las vías, el proceso se inicia con el análisis acústico de la cadena fónica, que permite establecer la correspondencia entre la información acústica y los fonemas que representa. A partir de este punto, las vías difieren. En la más habitual (vía 1), se identifican las palabras que tenemos almacenadas en el léxico auditivo (porque son palabras que ya hemos escuchado anteriormente, y las podemos reconocer). En el siguiente paso se recupera el significado de la palabra que tenemos en el sistema semántico, y de aquí, su forma ortográfica (léxico ortográfico), que se retiene a corto plazo en el sistema de grafemas, lo que nos permite escribirla. La segunda vía se activa cuando no conocemos el significado de una palabra (o bien nos dictan una pseudopalabra). En estos casos, no puede activarse el léxico auditivo (porque no habíamos escuchado nunca la palabra dictada) ni, en consecuencia, el sistema semántico. En su lugar, se activa el proceso de conversión acústico-fonológico que 
da un conjunto de fonemas. Estos fonemas son convertidos a grafemas mediante el mecanismo de conversión fonema a grafema ${ }^{7}$. Finalmente, la tercera vía (al igual que la primera) también activa el sistema semántico, pero en cambio no asume que se tiene la forma ortográfica almacenada en el léxico ortográfico, sino que activa el léxico fonológico (como si fuera a pronunciar la palabra), de modo que se convierten los fonemas en grafemas mediante un mecanismo específico. La existencia de estas rutas debe relacionarse con componentes lingüísticos de cada lengua, puesto que un mismo ejercicio de dictado no tiene la misma dificultad en todas las lenguas por las estructuras que se utilizan en la correspondencia de fonemas y símbolos escritos. En la teoría clásica Orthographic Depth Hypothesis (Frost, Katz \& Bentin, 1987), las lenguas se clasifican según la relación más o menos transparente u opaca entre sonidos y grafías. Por ejemplo, el español es más transparente que el inglés, que es una lengua considerada opaca. En teorías más recientes, como la de Ziegler y Goswami (2005, 2006), conocida como Grain Size Hypothesis, se establece un continuum entre relaciones fáciles y difíciles del procesamiento fonema-grafema. En esta teoría, se supone que las ortografías consistentes utilizan unidades lingüísticas pequeñas, mientras que las ortografías inconsistentes utilizan unidades mayores. Seymour, Aro \& Erskine (2003) consideran que además de la complejidad ortográfica, se debe considerar la complejidad estructural de las sílabas en una lengua determinada.

Como ejercicio escolar, son numerosos los autores que los defienden (Davis \& Rinvolucri, 1988; Montalvan, 1990; Kidd, 1992; Kiany \& Shiramiry, 2002; Cassany, 2004; García \& De la Torre, 2009) y, de hecho, los dictados siguen utilizándose extensivamente en las aulas tanto para la enseñanza de la L1 como de las lenguas extranjeras $^{8}$ (Cassany, 2004; García \& De la Torre, 2009; Generalitat de Catalunya, 2014). Por ejemplo, Montalvan (1990) señala 21 ventajas, que pueden agruparse en 4 categorías (Tabla 1 ).

A pesar de esto, en la actualidad los dictados no están bien considerados porque se asocian a prácticas 'tradicionales', 'pasadas de moda' y exclusivamente centradas en la ortografía. Cassany $(2004,231)$ afirma que los dictados suelen relacionarse con:

a) una práctica exclusiva de grado infantil y primario, relacionada con el aprendizaje de la lectoescritura

b) una concepción básicamente gramatical de la enseñanza lingüística, centrada en la memorización de reglas ortográficas

c) una visión reduccionista de la escritura, según la cual escribir consiste básicamente en "traducir" lo oral a lo gráfico o

d) un modelo didáctico conservador, basado en la exposición magistral y la ejercitación controlada, que entiende el error como una conducta mala que debe erradicarse, que fomenta la competitividad entre aprendices o que utiliza modelos lingüísticos literarios y alejados de la realidad y las necesidades del alumnado.

Cuando actúa esta vía es habitual cometer errores ortográficos, puesto que la correspondencia entre un fonema y su correspondiente grafema no es sistemática.

8 Hay que aclarar que existen diferencias sustanciales en los objetivos del uso de los dictados en la enseñanza de la lengua primera y de las lenguas extranjeras. Por ejemplo, en las lenguas extranjeras uno de los objetivos principales es entrenar la habilidad de la segmentación léxica (Field, 2003). Aun así, otros objetivos (como el aprendizaje de vocabulario o la práctica y evaluación de la ortografía) son compartidos. 
Tabla 1. Ventajas de los dictados, agrupadas (según el criterio de los autores). Los puntos se han reorganizado, aunque se citan literalmente.

\begin{tabular}{|c|c|}
\hline $\begin{array}{l}\text { Favorece la } \\
\text { integración de } \\
\text { las habilidades } \\
\text { lingüísticas } \\
\text { y potencia el } \\
\text { aprendizaje de la } \\
\text { lengua }\end{array}$ & $\begin{array}{l}\text { "1. Dictation can help develop all four language skills in an integrative way. } \\
\text { 2. As students develop their aural comprehension of meaning and also of } \\
\text { the relationship among segments of language, they are learning grammar. } \\
\text { 4. Practice in careful listening to dictation will be useful later on in note } \\
\text { taking exercises. } \\
\text { 5. Correcting dictation can lead to oral communication. } \\
\text { 6. Dictation can serve as an excellent review exercise. } \\
\text { 8. Dictation fosters unconscious thinking in the new language. } \\
\text { 20. Dictation can provide access to interesting texts, by introducing a topic, } \\
\text { for example, or summarizing it, as in a dicto-comp. } \\
\text { 21. Research has shown the learning to write down what you hear can } \\
\text { encourage the development of literacy. }\end{array}$ \\
\hline Trabaja la memoria & $\begin{array}{l}\text { 3. Dictation helps to develop short-term memory. Students practice } \\
\text { retaining meaningful phrases or whole sentences before writing them down. }\end{array}$ \\
\hline $\begin{array}{l}\text { Facilita la } \\
\text { organización de la } \\
\text { clase }\end{array}$ & $\begin{array}{l}\text { 10. Dictation involves the whole class, no matter how large it is. } \\
\text { 11. During and after the dictation, all the students are active. } \\
\text { 12. Correction can be done by the students. } \\
\text { 13. Dictation can be prepared for mixed ability groups. } \\
\text { 14. Dictation can be prepared for any level. } \\
\text { 15. The students, as well as the teacher, can get instant feedback (if the } \\
\text { exercise is corrected immediately). } \\
\text { 16. The dictation passage can (and should) be completely prepared in } \\
\text { advance. (It can also be taped.) } \\
\text { 17. Dictation can be administered quite effectively by an inexperienced } \\
\text { teacher. } \\
\text { 18. While dictating, the teacher can move about, giving individual attention. } \\
\text { 19. Dictation exercises can pull the class together, for example, during } \\
\text { those valuable first minutes. }\end{array}$ \\
\hline Motiva & $\begin{array}{l}\text { 7. Dictation is psychologically powerful and challenging. } \\
\text { 9. If the students do well, dictation is motivating." }\end{array}$ \\
\hline
\end{tabular}

Fuente: Montalvan, R. (1990). Dictation Updated: Guidelines for Teacher-Training Workshops. Recuperado de http://dosfan.lib.uic.edu/usia/E-USIA/education/engteaching/ intl/pubs/dictatn.htm (17/01/2017).

Sin embargo, las múltiples variantes de los dictados permiten realizar dictados significativos, creativos y dinámicos —e incluso divertidos-; basta "aprender a usar el dictado" (Cassany, 2004, 232). La variedad de dictados es enorme (solo el libro de 
Davis \& Rinvolucri, de 1988, aporta 69 variaciones distintas), por lo que remitimos al lector a la Tabla 1 y a la bibliografía para un listado más completo de ejercicios basados en el dictado. Como ejemplos de dictados poco habituales podemos citar el "dictado de secretaría" o dictogloss (Byrne, 1979; Davis \& Rinvolucri, 1988; Brinton, Snow \& Wesche, 1989; Wajnryb, 1990; Jacobs \& Small, 2003; Cassany, 2004; Vasiljevic, 2010), o el dictado de pared o running dictation (Davis \& Rinvolucri, 1988; Cassany, 2004). El primero consiste en dictar una única vez un texto a velocidad normal, de modo que los alumnos, habitualmente en pequeños grupos, puedan intentar reconstruir el texto a partir de sus recuerdos y las notas que hayan podido tomar. En este ejercicio lo fundamental no es reconstruir palabra a palabra el texto original, sino mantener las ideas principales del texto. El dictado de pared, por el contrario, tiene un carácter más competitivo: el texto se cuelga en un extremo de la clase o del patio, y los alumnos se agrupan por parejas. Uno toma el rol de mensajero y el otro de escriba (que se sitúa en el extremo opuesto de donde se encuentra el texto). El mensajero debe ir corriendo hacia el texto, leer un fragmento, memorizarlo (y fijándose específicamente en las dificultades léxicas, morfológicas, sintácticas u ortográficas) e ir hacia su compañero para dictárselo. Obviamente puede advertirle de los aspectos que presentan alguna dificultad específica. Gana la actividad la pareja que logra tener primero la versión correcta del texto original.

\section{Las representaciones de los dictados}

En este apartado se presentan los resultados de un cuestionario administrado a estudiantes del grado de Maestro en Educación Primaria y del doble grado de Maestro en Educación Primaria y Maestro en Educación Infantil en la Universidad de Gerona al inicio del curso 2016-2017. Se presentan en primer lugar (3.1) las características del cuestionario y de los informantes, el procedimiento de categorización de los datos, y los análisis estadísticos llevados a cabo. A continuación (3.2) se presentan y discuten los resultados obtenidos. Finalmente (3.3) se presentan algunas recomendaciones para los maestros en activo o en formación y para los expertos en didáctica de la lengua con el fin de mejorar la práctica de los dictados en la escuela primaria.

\subsection{Informantes, material y método}

El cuestionario fue administrado electrónicamente y debía contestarse fuera del horario lectivo, en el marco de la asignatura Lenguas 1 del grado de Maestro en Educación Primaria (que cursan también los estudiantes del doble grado). Esta asignatura está programada para segundo curso ${ }^{9}$. Los estudiantes que se matriculaban en la asignatura por segunda vez fueron descartados, puesto que ya recibieron formación explícita sobre el tema en el curso anterior.

Una vez completados los datos personales (nombre, vía de entrada a la universidad - vía bachillerato y pruebas de acceso, o bien ciclos formativos - y la especialidad), se presentaban las siguientes $\operatorname{preguntas}^{10}$ :

9 Los estudiantes de segundo curso no han cursado aún ningún periodo de prácticas, excepto dos semanas de observación en la escuela durante el primer curso.

10 El cuestionario era más extenso. No se incluye la información acerca de las otras preguntas porque no guardan relación con el tema de esta investigación. 
1. ¿Qué tipo de dictados conoces? Enuméralos y descríbelos brevemente.

2. ¿Para qué sirven los dictados en la Educación Primaria?

3. ¿Qué habilidades o aspectos lingüísticos ejercitan?

4. Como alumno de Primaria o de Secundaria, ¿te gustaba hacer dictados?

5. Cuando seas maestro de Primaria, ¿crees que los vas a utilizar? ¿De qué tipo?

6. ¿Con qué frecuencia deberían hacerse dictados en la Escuela Primaria?

7. ¿Deberían hacerse para todas las lenguas de la escuela (catalán, castellano y lengua extranjera) por igual?

Aunque se trataba de una actividad obligatoria y nominal, no fue evaluada, puesto que su objetivo era conocer las representaciones y las preconcepciones de los estudiantes hacia los dictados. En total 125 estudiantes (17 hombres y 108 mujeres) respondieron el cuestionario. La edad media de los informantes es de 20,2 años. Las instrucciones previas que recibieron hicieron hincapié en que no debían buscar información en bibliografía específica, puesto que el interés era conocer cuáles eran sus ideas previas (preconcepciones) acerca de los dictados (entre otros temas), de acuerdo con el modelo de formación de profesorado propuesto en Korthagen (2008).

Al tratarse de un cuestionario con algunas preguntas abiertas, fue necesario establecer categorías siguiendo una metodología sistemática (Altheide, 2002; Hargreaves, Lewis \& Speers, 2003). El procedimiento concreto se realizó en tres fases. En primer lugar, se analizó una muestra aleatoria de 20 cuestionarios, de cuyo análisis se establecían las categorías preliminares. A continuación, los dos autores analizaron independientemente otros 20 cuestionarios elegidos al azar, utilizando las categorías preliminares. La última fase consistió en la validación del procedimiento mediante el análisis de medida de acuerdo kappa de Cohen (kappa $=0,956$, Sig. $<0,001$ ), que demostró un alto grado de acuerdo en la categorización. El resto de cuestionarios fue analizado por solo uno de los investigadores.

Para las preguntas de respuesta abierta se establecieron las siguientes categorías descritas en las Tablas 2 y 3 . En la Tabla 2 se registran todos los dictados conocidos por los encuestados (con una descripción breve), así como sus variantes más significativas. Cabe destacar que la denominación de estos dictados difería según las respuestas, pero para simplificar se ha decidido utilizar la que, a nuestro criterio, es la más extendida (por ejemplo, el dictado 'tradicional' se denominó también 'literal' o 'verbatim'). La Tabla 3 muestra los usos identificados por los estudiantes. Aunque la pregunta era abierta, se ha procedido a categorizar si los estudiantes se referían a la práctica, a la evaluación o a ambas.

En un plano descriptivo, se ofrecen datos relativos a la frecuencia de cada categoría; complementariamente, también se ofrecen tablas de contingencia y pruebas de Chi-cuadrado con el fin de comprobar si existen diferencias estadísticamente significativas entre los grupos de estudiantes a los que les gustaban los dictados y los que no, por un lado, y entre los que como maestros afirman que van a utilizar los dictados en sus clases y los que no, por el otro.

Finalmente, con el fin de determinar si existe alguna relación entre las categorías establecidas se ha llevado a cabo un análisis de conglomerados jerárquico (mediante SPSS versión 19 para Windows). Este procedimiento permite agrupar las variables en función de su similaridad. Para este estudio, se ha utilizado el método de Ward para la conglomeración y la distancia euclídea al cuadrado como medida. Los datos se han estandarizado (transformándolos en un rango -1 a 1). 
Tabla 2. Categorías para la pregunta acerca del conocimiento de distintos tipos de dictados.

\begin{tabular}{|c|c|}
\hline Dictado 'tradicional'... & $\begin{array}{l}\text { El maestro dicta un texto desconocido por los alumnos, varias veces, } \\
\text { a la velocidad adecuada para que los alumnos puedan transcribirlo } \\
\text { literalmente. } \\
\text { Variantes: } \\
\text {...de frases } \\
\text {...de textos } \\
\text {...sin indicar la puntuación } \\
\text {....indicando la puntuación }\end{array}$ \\
\hline Dictado preparado... & $\begin{array}{l}\text { El maestro dicta un texto conocido por los alumnos, que han podido } \\
\text { analizarlo, estudiarlo con anterioridad. } \\
\text { Variantes: } \\
\text {...con espacios vacíos para completar (que representan alguna dificultad } \\
\text { ortográfica, como los pares } b / v, s / s s, \text { etc.) } \\
\text {...sin indicar la puntuación } \\
\text {...indicando la puntuación } \\
\text {...con errores que hay que detectar y corregir }\end{array}$ \\
\hline Por parejas & $\begin{array}{l}\text { Se trata de un dictado tradicional en el que el maestro no tiene ningún } \\
\text { papel activo, más allá de observar el desarrollo de la actividad y asistir a } \\
\text { los alumnos en caso de que sea necesario. Los alumnos se organizan por } \\
\text { parejas. Cada alumno dicta una parte del texto a su compañero. }\end{array}$ \\
\hline Fonético & $\begin{array}{l}\text { Se trata de un dictado de palabras con alguna dificultad fonética que } \\
\text { tiene relevancia en la escritura (por ejemplo, palabras con ese sorda o } \\
\text { sonora en catalán). }\end{array}$ \\
\hline $\begin{array}{l}\text { De imágenes, o dictado } \\
\text { mudo }\end{array}$ & $\begin{array}{l}\text { Se trata de un dictado en el que el maestro no oraliza el texto sino que } \\
\text { lo muestra a los alumnos mediante imágenes. Habitualmente se trata } \\
\text { de dictados de palabras, aunque para cursos más avanzados pueden } \\
\text { mostrarse viñetas que conformen una historia. }\end{array}$ \\
\hline Inventado & $\begin{array}{l}\text { Se trata de un dictado tradicional cuyo texto ha sido inventado por los } \\
\text { alumnos. }\end{array}$ \\
\hline De memoria & $\begin{array}{l}\text { Se trata de una variante del dictado preparado, pero los alumnos tienen } \\
\text { que memorizar el texto, puesto que nadie lo va a verbalizar. Es habitual } \\
\text { que el texto sea un poema u otro tipo de texto fácil de memorizar. }\end{array}$ \\
\hline $\begin{array}{l}\text { Con textos de los } \\
\text { alumnos }\end{array}$ & $\begin{array}{l}\text { Se trata de un dictado tradicional, pero el texto dictado ha sido } \\
\text { producido por algún alumno. }\end{array}$ \\
\hline Dibujado & $\begin{array}{l}\text { El maestro dicta un texto y los alumnos lo dibujan (sin escribir ninguna } \\
\text { palabra). }\end{array}$ \\
\hline Medio dictado & $\begin{array}{l}\text { Se trata de un ejercicio que combina el dictado tradicional y la } \\
\text { redacción. Habitualmente, el dictado consiste en la lectura de algunas } \\
\text { partes de un texto narrativo. Los alumnos deben complementar este } \\
\text { texto utilizando sus propias ideas. }\end{array}$ \\
\hline
\end{tabular}




\begin{tabular}{|l|l|}
\hline Cantado & $\begin{array}{l}\text { El texto dictado es una canción, que hay que transcribir, total o } \\
\text { parcialmente. Lo habitual es que la canción se repita entera (o por } \\
\text { estrofas) varias veces, porque no es posible dictarla a velocidad más } \\
\text { lenta o con pausas entre palabras. }\end{array}$ \\
\hline En grupo & $\begin{array}{l}\text { Ejercicio que combina la redacción sobre un tema determinado y el } \\
\text { dictado entre iguales. El profesor (o algún alumno) propone un tema, } \\
\text { una frase inacabada..., y los alumnos escriben algunas frases sobre la } \\
\text { propuesta. Después, se las dictan entre ellos, en pequeños grupos. }\end{array}$ \\
\hline Gramatical & $\begin{array}{l}\text { Dictado tradicional en el que el maestro propone alguna transformación } \\
\text { (basada en algún aspecto gramatical, como el género, el número, el } \\
\text { tiempo, etc.). A continuación, dicta el texto, que los estudiantes deben } \\
\text { copiar directamente con la transformación ya aplicada. }\end{array}$ \\
\hline Dictado de consulta & $\begin{array}{l}\text { Dictado tradicional en el que los alumnos disponen de tiempo para } \\
\text { buscar en el diccionario las palabras de cuya ortografía dudan. }\end{array}$ \\
\hline De lengua inversa & $\begin{array}{l}\text { Dictado tradicional en el que el maestro dicta en una lengua y los niños } \\
\text { transcriben en otra. Típicamente, las parejas de lenguas son el catalán y } \\
\text { el castellano, puesto que son las que los alumnos dominan mejor. }\end{array}$ \\
\hline Colectivo & $\begin{array}{l}\text { Dictado tradicional que posteriormente se comenta en grupos pequeños } \\
\text { para comparar versiones antes de entregar una versión final. }\end{array}$ \\
\hline
\end{tabular}

Tabla 3. Categorías para la pregunta acerca de la utilidad de los dictados. Para cada categoría, se ha marcado si los estudiantes han manifestado que el contenido o habilidad se ejercita mediante el dictado, si se puede evaluar, o ambos.

\begin{tabular}{|l|c|c|}
\hline & Para practicar & Para evaluar \\
\hline Ortografía & $\sqrt{ }$ & $\sqrt{ }$ \\
\hline Gramática & $\sqrt{ }$ & \\
\hline Puntuación & $\sqrt{ }$ & \\
\hline Vocabulario & $\sqrt{ }$ & \\
\hline Lectura (comprensión lectora) & $\sqrt{ }$ & $\sqrt{ }$ \\
\hline Escucha (comprensión oral) & $\sqrt{ }$ & \\
\hline Escritura & $\sqrt{ }$ \\
\hline Caligrafía & $\sqrt{ }$ & $\sqrt{ }$ \\
\hline Pronunciación & $\sqrt{ }$ \\
\hline Memoria & $\sqrt{ }$ \\
\hline Atención & $\sqrt{ }$ & \\
\hline Uso del diccionario & & \\
\hline
\end{tabular}




\subsection{Resultados}

Globalmente, los estudiantes mencionaron en total 16 tipos de dictados distintos (en ocasiones con variantes, como hemos visto en la Tabla 2). La Figura 2 muestra el porcentaje de conocimiento de cada tipo de dictado por parte de los alumnos. Puede observarse que los dictados 'tradicionales' son los más conocidos (el 98,4\% de los estudiantes los conocen ${ }^{11}$ ); el segundo grupo de dictados más conocido es el preparado $(60,8 \%)$, seguido por el dictado fonético (44\%). A mayor distancia encontramos el resto de dictados. De estos, cinco fueron mencionados por un único estudiante (los que representan el 0,8\%), y el que mayor porcentaje de conocimiento representa (el dictado por parejas) fue mencionado por solo 11 estudiantes. Así, podría afirmarse que la mayoría de estudiantes solo conocen entre dos y tres tipos de dictados diferentes y solo unos pocos tienen conocimiento de una mayor variedad.

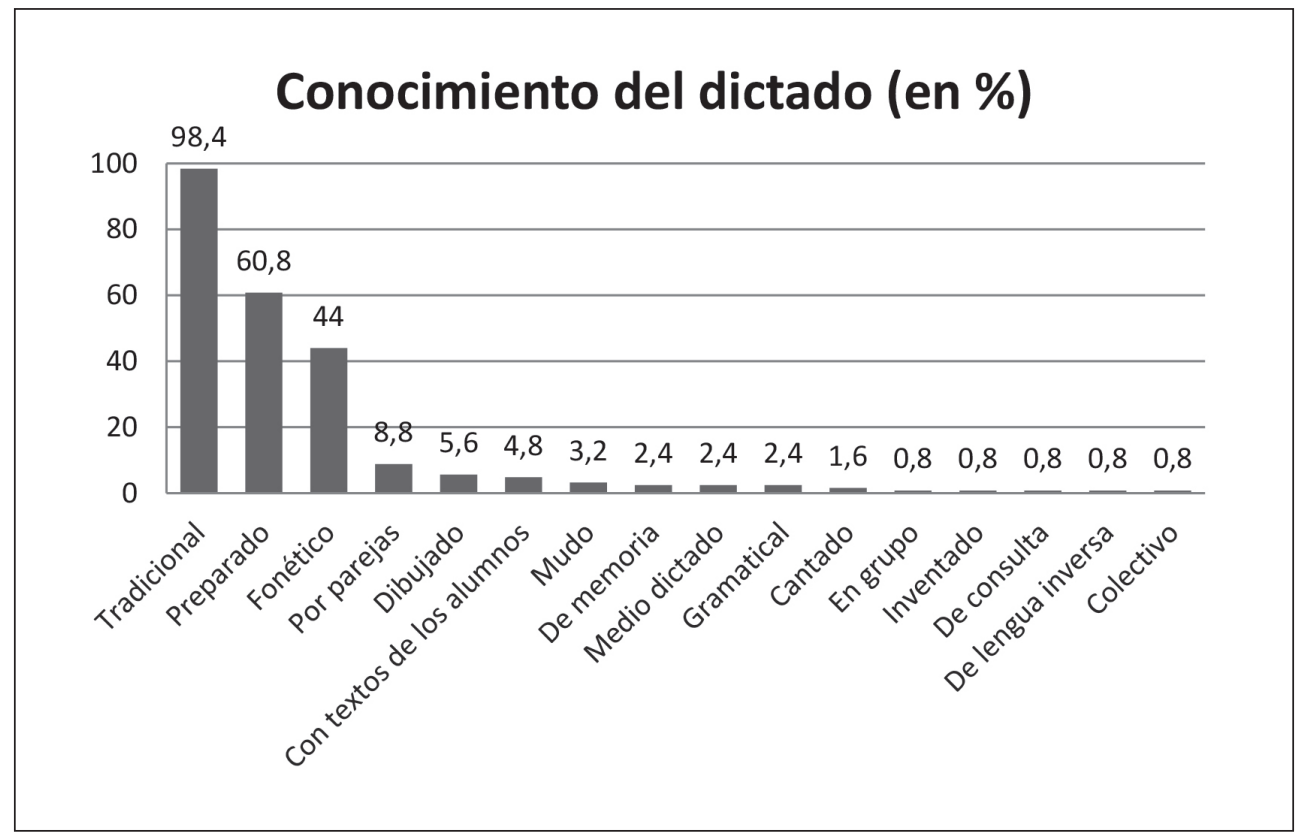

Figura 2. Porcentaje de conocimiento de cada tipo de dictado.

De hecho, los estudiantes, sin tener en cuenta las variaciones de un mismo tipo de dictado, mencionaron una media de 2,38 dictados diferentes (con una desviación estándar de 1,014) y con una única respuesta en el peor de los casos, y cinco en el mejor. Estos datos demuestran que, a pesar de todos los recursos a disposición de los maestros y profesores, la mayoría de docentes se ciñe a los dictados más tradicionales, por lo que no aprovechan las posibilidades que brindan los demás tipos.

11 Solo dos estudiantes no los mencionan. Es muy probable, sin embargo, que no los mencionen por descuido, puesto que la práctica de los dictados está muy extendida en la mayoría de escuelas (e institutos de Secundaria). 
La Figura 3 muestra las respuestas a la pregunta sobre la utilidad de los dictados, en porcentaje. Observamos que la gran mayoría $(94,4 \%)$ manifiestan que sirven para mejorar o evaluar la ortografía, que es el uso tradicional que le dan la mayoría de maestros. Sin embargo, también hay otras respuestas con porcentajes superiores al 20\%: para trabajar la comprensión oral $(25,6 \%)$, la gramática $(24,8 \%)$, el vocabulario $(20,8 \%)$ y la escritura $(20 \%)$. El resto de utilidades (ver Figura 3) son mencionadas en un menor número de casos. Por ejemplo, el uso del diccionario fue mencionado por un solo estudiante (el mismo que hizo referencia al dictado de consulta).

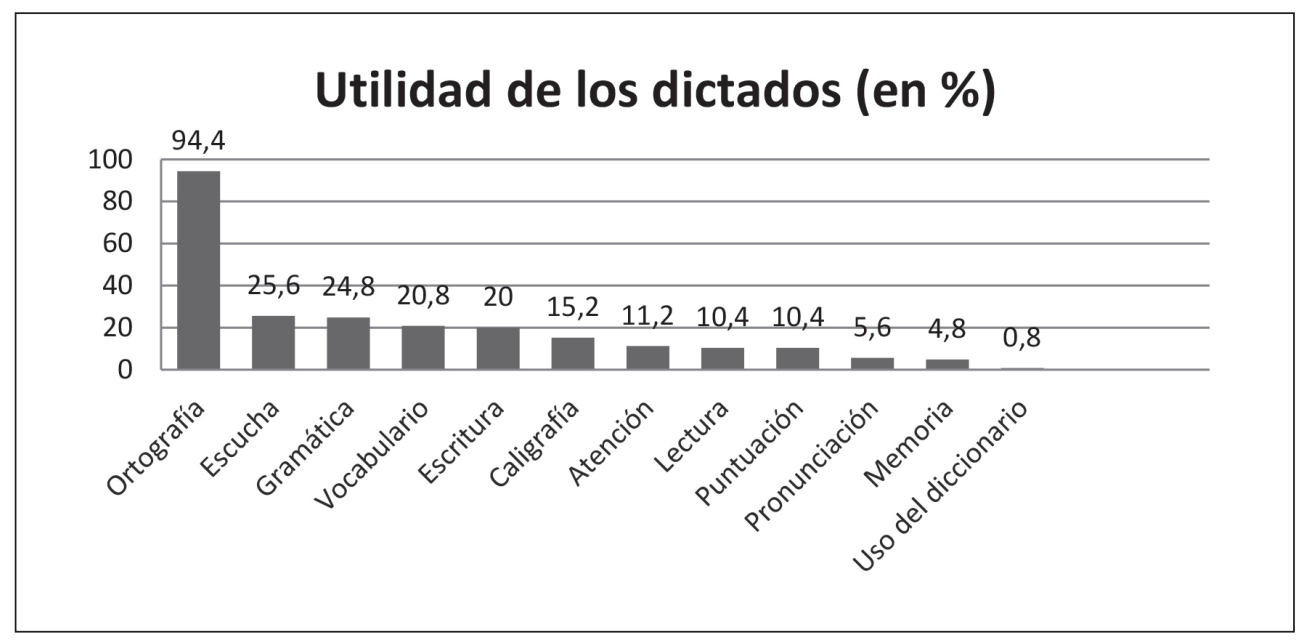

Figura 3. Representaciones de la utilidad de los dictados. Porcentaje de respuestas.

A pesar del escaso número de dictados conocidos por los estudiantes, la mayoría $(62,2 \%)$ manifiestan que, como alumnos de Primaria, les gustaba hacer dictados, frente al 37,8\% que no. Aun así, la práctica totalidad de los alumnos encuestados afirman que cuando tengan la responsabilidad de decidir si llevar a cabo esta actividad (como maestros de Primaria) harán este ejercicio regularmente (96\%). Solo 4 estudiantes $(3,2 \%)$ manifestaron que como máximo harían algún dictado esporádicamente. La frecuencia con la que se deberían hacer dictados se reparte prácticamente en partes iguales entre los partidarios de hacer un dictado a la semana $(34,2 \%)$, de hacer menos de uno $(32,9 \%)$ o más de uno $(32,9 \%)$ semanales. Finalmente, la mayoría de los estudiantes $(71,1 \%)$ manifiesta que los dictados deberían hacerse en la misma proporción entre todas las lenguas estudiadas en la escuela (en el caso de las escuelas catalanas, el catalán, el español y la lengua extranjera). El 12,2\% cree que las lenguas más débiles (la lengua extranjera, y en ocasiones el catalán o el español, según las características sociolingüísticas de los alumnos) deberían reforzarse con una mayor proporción de dictados. La postura contraria (hacer más dictados en las lenguas más fuertes: catalán, castellano o ambas) es defendida por el 16,7\% de los encuestados. Los argumentos que aportan tienen que ver con el hecho de que el dictado sirve para profundizar en el aprendizaje de la lengua, por lo que si no se tiene un dominio suficiente de la lengua el dictado puede llegar a ser contraproducente. 
Finalmente, se ha estudiado la relación entre los dictados conocidos por los estudiantes y su utilidad. Para ello, se ha realizado un análisis de conglomerados jerárquico, cuyo dendrograma ha permitido discriminar entre dos conjuntos de variables principales (Figura 4). Por un lado, se asocian claramente los dictados tradicionales y los dictados preparados con el uso para practicar y evaluar la ortografía. Este grupo se corresponde con los dictados más conocidos por los estudiantes (a gran diferencia del resto) vinculados a la práctica y evaluación de la ortografía. Por el otro lado, el dendograma agrupa el resto de usos (Figura 3) y de dictados (claramente menos conocidos, como hemos visto en la Figura 2). Las respuestas de los estudiantes y su análisis relacionado parecen indicar que su experiencia con el dictado en las escuelas e institutos sigue estrechamente vinculada a los dictados más tradicionales (el literal y el dictado preparado) y al uso vinculado con la enseñanza y evaluación de la ortografía. Finalmente, existe cierta conciencia -aún no mayoritaria- de que los dictados son ejercicios que van más allá de la práctica y evaluación de la ortografía.

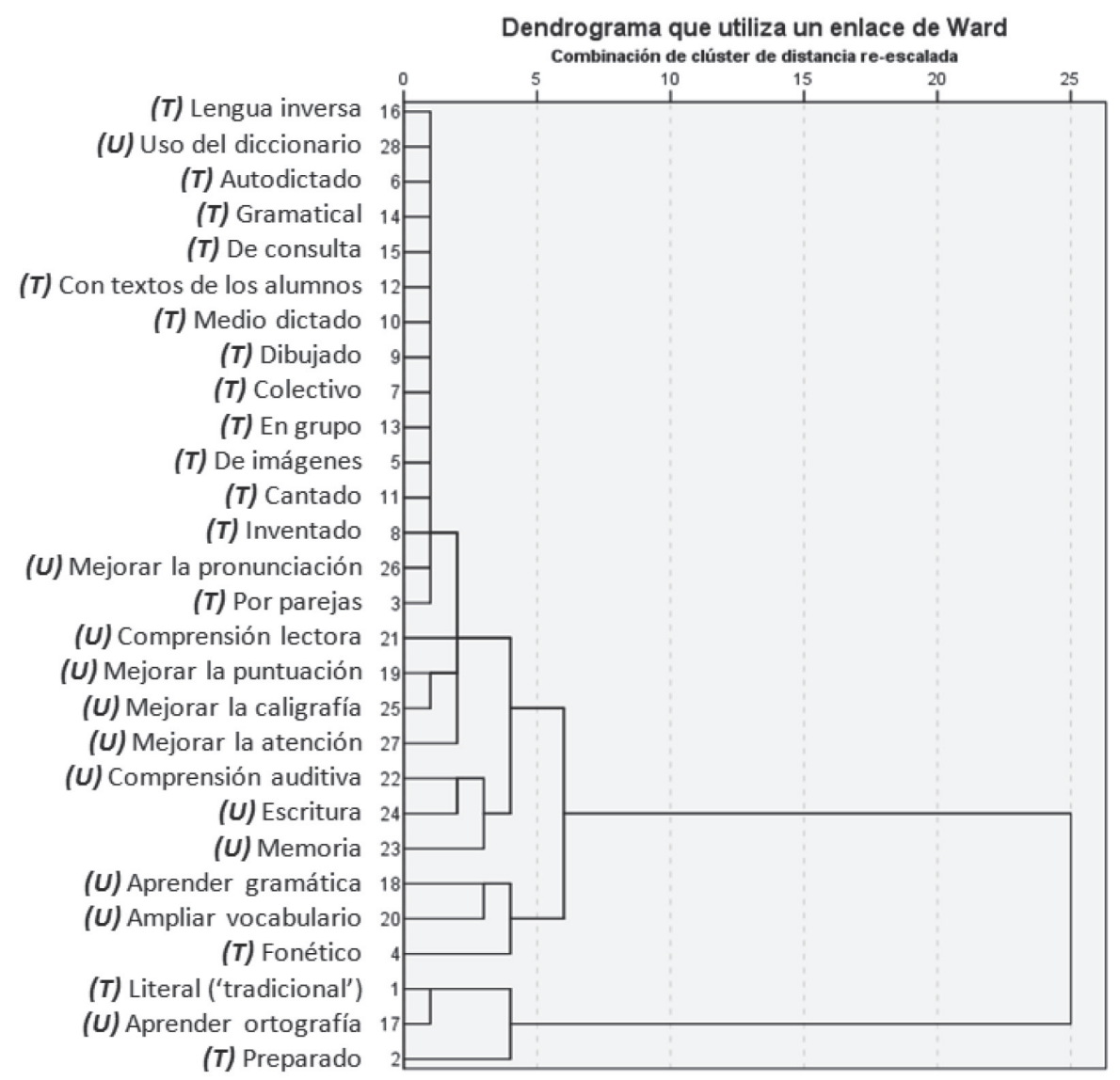

(T) Lengua inversa (U) Uso del diccionario

(T) Autodictado

(T) Gramatical

(T) De consulta de los alum

(T) Colectivo

(T) En grupo

(T) Cantado 1 (T)

(T) Inventado

(T) Por parejas

Comprensión lector

(U) Mejorar la atención

(U) Comprensión auditiva

(U) Escritura

(U) Memoria

(U) Aprender gramática

(U) Ampliar vocabulario

(T) Fonético

( $T$ ) Literal ('tradicional')

(T) Preparado

Figura 3. Dendrograma que muestra los grupos de variables referentes a los tipos de dictados (T) y a los usos (U). 


\section{Conclusiones y recomendaciones}

Los resultados de este estudio han mostrado que los estudiantes encuestados conocen, en general, pocos dictados (citan una media de 2,38 tipos distintos, de entre los múltiples tipos existentes) y que mayoritariamente citan solo los tradicionales (el dictado literal y el preparado). Por tanto, sería recomendable intensificar el conocimiento y la práctica de otros tipos de dictado para la formación de maestros o para los maestros en ejercicio, por ejemplo siguiendo los tipos que se describen en Cassany (2004).

Otro de los elementos que se tienen que considerar en la formación inicial y continua de maestros es la necesidad de que los futuros maestros adquieran las competencias que deberían capacitarlos para integrar la práctica de los dictados dentro de la línea pedagógica general, y la propuesta metodológica de enseñanza y aprendizaje de las lenguas del centro donde trabajan. Si se hace una apuesta por el uso de los dictados, por un lado se deben superar los prejuicios que habitualmente se asocian a este tipo de actividad, como (a) el uso exclusivo de los dictados para el aprendizaje de las reglas ortográficas, (b) la idea de que el dictado es una actividad dirigida que solo se puede relacionar con un modelo didáctico conservador o (c) que implica una actividad dirigida por el docente y reproducida por el aprendiz (Cassany, 2004). Por el otro lado, se deben tener en cuenta las ventajas que ofrecen los dictados según la bibliografía (Montalvan, 1990): son actividades que potencian todas las habilidades lingüísticas, son motivadoras, ayudan a la organización de la clase y ayudan a ejercitar la memoria. Así pues, el planteamiento de los dictados debe tener en cuenta por lo menos algunos de los siguientes aspectos:

1. Deben tener objetivos de aprendizaje concretos, en relación con alguna de las habilidades lingüísticas o generales (escucha, escritura, creatividad, memoria) y no solo con la ortografía.

2. Pueden utilizar técnicas de ludificación (concursos, puntos, premios, competición, recompensas).

3. Deberían alternar agrupaciones diversas de alumnos (trabajo individual, por parejas, en grupos).

4. Deberían promover la participación de los alumnos en la revisión y evaluación.

5. Deberían permitir que los alumnos establecieran relaciones con otras prácticas orales y reflexivas, de modo que les fuera posible detectar problemas y reflexionar sobre ellos.

Estos aspectos pueden dar sentido al dictado como herramienta situada en metodologías activas, más acorde a un modelo de enseñanza y aprendizaje basado en la adquisición de competencias, no solo de conocimientos. Siguiendo con esta idea de integrar los dictados en un modelo coherente para el aprendizaje y enseñanza de la lengua, si en un centro se decide optar por actividades que utilizan mayoritariamente textos reales y significativos, los dictados también deberían contribuir a esta línea y no aparecer como una actividad aislada sin relación con las demás. Por otra parte, si se utiliza mucho el juego como elemento motivador en general, también el dictado debe formar parte de esta orientación.

El hecho de tener en cuenta la inclusión del dictado en una línea definida de aprendizaje de lenguas coherente también lleva a plantear la necesaria coordinación de los maes- 
tros que se ocupan de las distintas lenguas y de otras materias (con el fin de hacer dictados más significativos, se podrían integrar los dictados en asignaturas no necesariamente lingüísticas, por ejemplo, dictando textos con contenidos clave de materias no lingüísticas). Asimismo, se podrían distribuir los aprendizajes que se asocian a los dictados en las distintas asignaturas de lengua del currículum. Más allá de los aprendizajes lingüísticos, también puede haber actividades relacionadas con otros ámbitos en los cuales sea útil el uso de un dictado (como por ejemplo dictar notas informativas para las familias).

Por último, el uso de los dictados puede adaptarse perfectamente a las formas de comunicación actuales. No es habitual realizar dictados en el ordenador, cuando probablemente las prácticas reales relacionadas con el dictado se realizan a través de esta herramienta. Pero, aunque la práctica del dictado no sea para finalidades reales, sino puramente académicas, es conveniente combinar el dictado manuscrito con el dictado en el ordenador (junto al uso de las herramientas lingüísticas asociadas, como el verificador ortográfico y la formatación del texto). Sea como sea, es necesario que los futuros maestros y los maestros en ejercicio dominen la actividad que han decidido realizar, en este caso el dictado, puedan definir para qué objetivos didácticos la están utilizando y sean capaces de integrarla en el modelo de enseñanza y aprendizaje que están implementando

\section{Bibliografía}

Altheide, David L. (2002). Creating Fear: News and the Construction of Crisis, Hawthorne, Nueva York, Aldine de Guyter, doi:https://doi.org/10.5860/choice.40-0726.

Brinton, Donna, M. A. Snow \& M. B. Wesche (1989). Content-Based Second Language Instruction. Nueva York: Newbury House Publishers.

Byrne, Donn (1979): Teaching Writing Skills, Londres, Longman.

Cassany, Daniel (2004). "El dictado como tarea comunicativa", en Tabula Rasa, 2, 229-250.

Cuetos, Fernando (2011). Psicología de la escritura ( $8^{a}$ edición). Las Rozas, Madrid: Wolters Kluwer España.

Davis, Paul \& M. Rinvolucri (1988). Dictation. New Methods, New Possibilities. Cambridge: Cambridge University Press.

Field, John (2003). "Promoting perception: lexical segmentation in L2 listening", en ELT Jorunal, 57(4), 325-334.

Frost, Ram, L. Katz \& S. Bentin (1987). "Strategies for Visual Word Recognition and Ortographical Depth: a Multilingual Comparison", en Journal of Experimental Psychology: Human Perception and Performance, 13, 104-115.

García, Miguel \& N. de la Torre (2009). "Deconstruyendo dictados”, en Suplementos MarcoELE, 9, 1-11.

Generalitat de Catalunya (2014). Orientacions pràctiques per a la millora de l'ortografia. Barcelona: Generalitat de Catalunya, Departament d'Ensenyament, Direcció General d'Educació Secundària Obligatòria i Batxillerat.

Hargreaves, Ian, J. Lewis \& T. Speers (2003). Towards a Better Map: Science, the Public and the Media. Cardiff: Cardiff University.

Irvine, Patricia, P. Atai \& J. W. Oller (1974). "Cloze, Dictation, and the Test of English as a Foreign Language”, en Language Learning, 24, 245-52.

Jacobs, George \& J. Small (2003). "Combining Dictogloss and Cooperative Learning to Promote Language Learning”, en The Reading Matrix, 3(1), 1-15. 
Joynes, Edward S. (1900). "Dictation and Composition in Modern Language Teaching”, en $P M L A, 15, \mathrm{xxv}$-xxxi.

Kiany, G. Reza \& E. Shiramiry (2002). "The Effect of Frequent Dictation on the Listening Comprehension Ability of Elementary EFL Learners", en TESL Canada Journal/Revue TESL du Canada, 20(1), 57-63, doi:https://doi.org/10.18806/tesl.v20i1.938.

Kidd, Richard (1992). “Teaching ESL Grammar through Dictation”, en TESL Canada Journal/Revue TESL du Canada, 10(1), 49-61, doi:https://doi.org/10.18806/tesl.v10i1.611.

Korthagen, Fred A. J. (2008). Linking Practice and Theory. The Pedagogy of Realistic Teacher Education, Abingdon, Routledge.

Lado, Robert (1961). Language Testing: The Construction and Use of Language Tests. Nueva York: McGraw-Hill.

Montalvan, Ruth G. (1990). Dictation Updated: Guidelines for Teacher-Training Workshops. http://dosfan.lib.uic.edu/usia/E-USIA/education/engteaching/intl/pubs/dictatn.htm [Consulta: 17 de enero de 2017].

Oller, John W. (1971). "Dictation as a Devide for Testing Foreign Language Proficency”, en English Language Teaching, 25, 254-59.

Richards, Jack C. \& T. Rodgers (2014). Approaches and Methods in Language Teaching, Cambridge, Cambridge University Press (3a edición), doi: https://doi.org/10.1017/ cbo9780511667305.

Sánchez, Aquilino (2009). La enseñanza de idiomas en los últimos cien años. Métodos y enfoque. Madrid: SGEL.

Seymour, Philip H. K., M. Aro \& J. M. Erskine (2003). "Foundation Literacy Acquisition in European Orthographies", en British Journal of Psychology, 94, 143-174. doi: doi:https:// doi.org/10.1348/000712603321661859.

Stansfield, Charles W. (1985). A History of Dictation in Foreign Language Teaching and Testing, en The Modern Language Journal, 69(2), 121-128, doi:https://doi.org/10.2307/326501.

Valette, Rebecca M. (1964). The Use of the Dictée in the French Language Classroom, en Modern Language Journal, 48, 431-34, doi:https://doi.org/10.2307/322627.

Vasiljevic, Zorana (2010). "Dictogloss as an Interactive Method of Teaching Listening Comprehension to L2 Learners", en English Language Teaching, 3(1), 41-52, doi:https://doi. org/10.5539/elt.v3n1p41.

Wajnryb, Ruth (1990). Grammar Dictation, Oxford, Oxford University Press.

Ziegler, Johannes \& U. Goswami (2005): "Reading Acquisition, Developmental Dyslexia and Skilled Readins Across Languages: a Psycholinguistic Grain Size Theory", en Psychological Bulletin, 13, 3-29.

Ziegler, Johannes \& U. Goswami (2006). "Becoming Literate in Different Languages: Similar Problems, Different Solutions", en Developmental Science, 9(5), 429-436. 